\title{
A highly-simplified and inexpensive MALDI-TOF mass spectrometry sample preparation with broad applicability to microorganisms, plants, and insects
}

Michael A. Reeve, Alan G. Buddie, Kathryn M. Pollard, Sonal Varia, Marion K. Seier, Lisa C. Offord, Matthew J.W. Cock

Table S1. Spectral similarity analysis for Aspergillus samples.

(A) Spectral similarity analysis - simplified protocol

\begin{tabular}{|c|c|c|c|c|c|c|c|c|c|c|c|c|c|c|}
\hline & 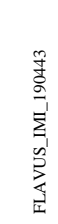 & 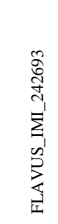 & 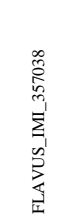 & 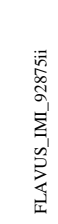 & 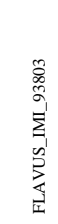 & 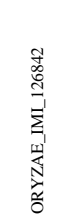 & 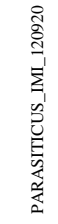 & 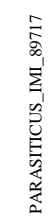 & 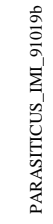 & 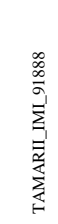 & 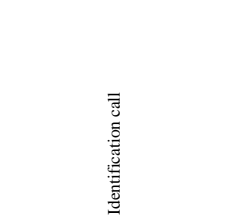 & 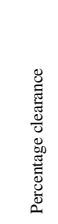 & 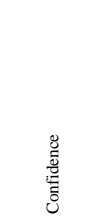 & 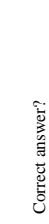 \\
\hline FLAVUS_IMI_190443_PLATE_1_TEST & 429 & 140 & 21 & 151 & 163 & 159 & 11 & 18 & 11 & 5 & FLAVUS_IMI_190443 & 62 & High & Yes \\
\hline FLAVUS_IMI_190443_PLATE_2_TEST & 420 & 152 & 24 & 146 & 146 & 155 & 10 & 15 & 10 & 2 & FLAVUS_IMI_190443 & 63 & High & Yes \\
\hline FLAVUS_IMI_242693_PLATE_1_TEST & 145 & 470 & 36 & 320 & 140 & 146 & 3 & 10 & 7 & 3 & FLAVUS_IMI_242693 & 32 & High & Yes \\
\hline FLAVUS_IMI_242693_PLATE_2_TEST & 168 & 514 & 40 & 337 & 139 & 196 & 3 & 10 & 7 & 2 & FLAVUS_IMI_242693 & 34 & High & Yes \\
\hline FLAVUS_IMI_357038_PLATE_1_TEST & 24 & 47 & 381 & 39 & 15 & 20 & 6 & 1 & 2 & 3 & FLAVUS_IMI_357038 & 88 & High & Yes \\
\hline FLAVUS_IMI_357038_PLATE_2_TEST & 36 & 65 & 474 & 74 & 32 & 43 & 11 & 2 & 8 & 2 & FLAVUS_IMI_357038 & 84 & High & Yes \\
\hline FLAVUS_IMI_92875ii_PLATE_1_TEST & 186 & 292 & 35 & 507 & 157 & 115 & 3 & 22 & 8 & 2 & FLAVUS_IMI_92875ii & 42 & High & Yes \\
\hline FLAVUS_IMI_92875ii_PLATE_2_TEST & 87 & 283 & 60 & 387 & 89 & 97 & 3 & 7 & 4 & 1 & FLAVUS_IMI_92875ii & 27 & High & Yes \\
\hline FLAVUS_IMI_93803_PLATE_1_TEST & 119 & 131 & 12 & 148 & 518 & 157 & 9 & 10 & 19 & 2 & FLAVUS_IMI_93803 & 70 & High & Yes \\
\hline FLAVUS_IMI_93803_PLATE_2_TEST & 104 & 138 & 10 & 163 & 476 & 137 & 4 & 5 & 8 & 2 & FLAVUS_IMI_93803 & 66 & High & Yes \\
\hline ORYZAE_IMI_126842_PLATE_1_TEST & 113 & 208 & 50 & 143 & 119 & 343 & 8 & 7 & 5 & 6 & ORYZAE_IMI_126842 & 39 & High & Yes \\
\hline ORYZAE_IMI_126842_PLATE_2_TEST & 174 & 204 & 22 & 124 & 138 & 444 & 12 & 11 & 11 & 2 & ORYZAE_IMI_126842 & 54 & High & Yes \\
\hline PARASITICUS_IMI_120920_PLATE_1_TEST & 10 & 4 & 3 & 5 & 12 & 9 & 207 & 107 & 183 & 2 & PARASITICUS_IMI_120920 & 12 & Reasonable & Yes \\
\hline PARASITICUS_IMI_120920_PLATE_2_TEST & 8 & 7 & 5 & 10 & 14 & 13 & 450 & 110 & 292 & 2 & PARASITICUS_IMI_120920 & 35 & High & Yes \\
\hline PARASITICUS_IMI_89717_PLATE_1_TEST & 20 & 12 & 4 & 22 & 9 & 22 & 102 & 570 & 106 & 4 & PARASITICUS_IMI_89717 & 81 & High & Yes \\
\hline PARASITICUS_IMI_89717_PLATE_2_TEST & 16 & 6 & 1 & 13 & 12 & 9 & 53 & 160 & 81 & 4 & PARASITICUS_IMI_89717 & 49 & High & Yes \\
\hline PARASITICUS_IMI_91019b_PLATE_1_TEST & 8 & 11 & 9 & 11 & 17 & 9 & 289 & 96 & 531 & 3 & PARASITICUS_IMI_91019b & 46 & High & Yes \\
\hline PARASITICUS_IMI_91019b_PLATE_2_TEST & 7 & 6 & 3 & 9 & 7 & 7 & 274 & 123 & 430 & 2 & PARASITICUS_IMI_91019b & 36 & High & Yes \\
\hline TAMARII_IMI_91888_PLATE_1_TEST & 3 & 2 & 1 & 4 & 3 & 5 & 3 & 4 & 2 & 232 & TAMARII_IMI_91888 & 98 & High & Yes \\
\hline TAMARII_IMI_91888_PLATE_2_TEST & 1 & 1 & 2 & 1 & 1 & 2 & 4 & 2 & 2 & 306 & TAMARII_IMI_91888 & 99 & High & Yes \\
\hline
\end{tabular}


(B) Spectral similarity analysis - full-extraction controls

\begin{tabular}{|c|c|c|c|c|c|c|c|c|c|c|c|c|c|}
\hline & 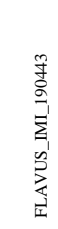 & 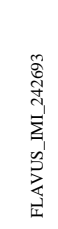 & 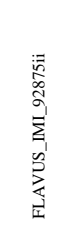 & 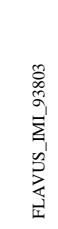 & 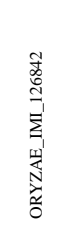 & 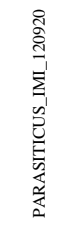 & 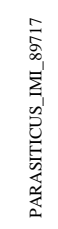 & 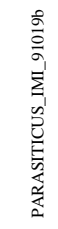 & 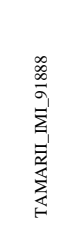 & 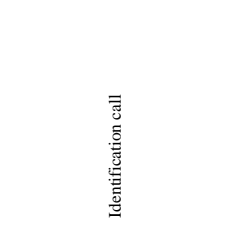 & 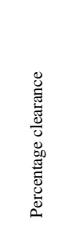 & 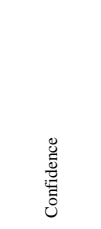 & 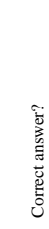 \\
\hline FLAVUS_IMI_190443_PLATE_1_TEST & 330 & 174 & 123 & 137 & 104 & 19 & 37 & 14 & 5 & FLAVUS_IMI_190443 & 21 & High & Yes \\
\hline FLAVUS_IMI_190443_PLATE_2_TEST & 320 & 153 & 120 & 153 & 172 & 14 & 37 & 13 & 3 & FLAVUS_IMI_190443 & 11 & Reasonable & Yes \\
\hline FLAVUS_IMI_242693_PLATE_1_TEST & 112 & 511 & 256 & 189 & 157 & 19 & 35 & 13 & 5 & FLAVUS_IMI_242693 & 50 & High & Yes \\
\hline FLAVUS_IMI_242693_PLATE_2_TEST & 187 & 454 & 265 & 204 & 214 & 20 & 39 & 20 & 5 & FLAVUS_IMI_242693 & 42 & High & Yes \\
\hline FLAVUS_IMI_92875ii_PLATE_1_TEST & 116 & 302 & 352 & 145 & 163 & 24 & 40 & 27 & 8 & FLAVUS_IMI_92875ii & 14 & Reasonable & Yes \\
\hline FLAVUS_IMI_92875ii_PLATE_2_TEST & 96 & 333 & 322 & 166 & 153 & 23 & 32 & 17 & 5 & FLAVUS_IMI_242693 & 3 & Low & No \\
\hline FLAVUS_IMI_93803_PLATE_2_TEST & 107 & 129 & 100 & 513 & 144 & 14 & 45 & 15 & 6 & FLAVUS_IMI_93803 & 72 & High & Yes \\
\hline ORYZAE_IMI_126842_PLATE_1_TEST & 152 & 176 & 164 & 174 & 485 & 21 & 73 & 24 & 3 & ORYZAE_IMI_126842 & 64 & High & Yes \\
\hline ORYZAE_IMI_126842_PLATE_2_TEST & 160 & 145 & 145 & 217 & 428 & 35 & 56 & 20 & 4 & ORYZAE_IMI_126842 & 49 & High & Yes \\
\hline PARASITICUS_IMI_120920_PLATE_1_TEST & 32 & 27 & 32 & 31 & 28 & 472 & 93 & 409 & 7 & PARASITICUS_IMI_120920 & 13 & Reasonable & Yes \\
\hline PARASITICUS_IMI_120920_PLATE_2_TEST & 44 & 37 & 33 & 25 & 40 & 482 & 113 & 374 & 6 & PARASITICUS_IMI_120920 & 22 & High & Yes \\
\hline PARASITICUS_IMI_89717_PLATE_1_TEST & 37 & 46 & 32 & 55 & 63 & 60 & 499 & 73 & 6 & PARASITICUS_IMI_89717 & 85 & High & Yes \\
\hline PARASITICUS_IMI_89717_PLATE_2_TEST & 42 & 40 & 36 & 56 & 71 & 81 & 532 & 97 & 8 & PARASITICUS_IMI_89717 & 82 & High & Yes \\
\hline PARASITICUS_IMI_91019b_PLATE_1_TEST & 42 & 46 & 35 & 28 & 45 & 425 & 129 & 460 & 5 & PARASITICUS_IMI_91019b & 8 & Low & Yes \\
\hline PARASITICUS_IMI_91019b_PLATE_2_TEST & 31 & 39 & 47 & 37 & 49 & 378 & 131 & 549 & 4 & PARASITICUS_IMI_91019b & 31 & High & Yes \\
\hline
\end{tabular}


Table S2. Identification results for Crassula samples.

\begin{tabular}{|c|c|c|c|c|}
\hline Blind-test sample & Best match & Bruker Score & Second-best match & Bruker score \\
\hline CRASSULA_BLIND_TEST_01 & CRASSULA_AQUATICA_REF_5 & 2.683 & CRASSULA_AQUATICA_REF_2 & 2.640 \\
\hline CRASSULA_BLIND_TEST_02 & CRASSULA_HELMSII_FLINDERS_ISLAND_REF_6 & 2.584 & CRASSULA_HELMSII_FLINDERS_ISLAND_REF_4 & 2.556 \\
\hline CRASSULA_BLIND_TEST_03 & CRASSULA_HELMSII_VICTORIA_REF_4 & 2.655 & CRASSULA_HELMSII_VICTORIA_REF_3 & 2.551 \\
\hline CRASSULA_BLIND_TEST_04 & CRASSULA_AQUATICA_REF_5 & 2.748 & CRASSULA_AQUATICA_REF_3 & 2.725 \\
\hline CRASSULA_BLIND_TEST_05 & CRASSULA_HELMSII_BELGIUM_REF_5 & 2.747 & CRASSULA_HELMSII_BELGIUM_REF_4 & 2.666 \\
\hline CRASSULA_BLIND_TEST_06 & CRASSULA_HELMSII_UK_REF_1 & 2.700 & CRASSULA_HELMSII_UK_REF_6 & 2.564 \\
\hline CRASSULA_BLIND_TEST_07 & CRASSULA_AQUATICA_REF_5 & 2.764 & CRASSULA_AQUATICA_REF_4 & 2.730 \\
\hline CRASSULA_BLIND_TEST_08 & CRASSULA_HELMSII_UK_REF_4 & 2.717 & CRASSULA_HELMSII_UK_REF_1 & 2.630 \\
\hline CRASSULA_BLIND_TEST_09 & CRASSULA_HELMSII_VICTORIA_REF_5 & 2.719 & CRASSULA_HELMSII_VICTORIA_REF_3 & 2.637 \\
\hline CRASSULA_BLIND_TEST_10 & CRASSULA_HELMSII_FLINDERS_ISLAND_REF_1 & 2.472 & CRASSULA_HELMSII_FLINDERS_ISLAND_REF_3 & 2.237 \\
\hline CRASSULA_BLIND_TEST_11 & CRASSULA_HELMSII_FLINDERS_ISLAND_REF_6 & 2.685 & CRASSULA_HELMSII_FLINDERS_ISLAND_REF_2 & 2.626 \\
\hline CRASSULA_BLIND_TEST_12 & CRASSULA_HELMSII_BELGIUM_REF_1 & 2.705 & CRASSULA_HELMSII_BELGIUM_REF_5 & 2.687 \\
\hline CRASSULA_BLIND_TEST_13 & CRASSULA_HELMSII_BELGIUM_REF_5 & 2.691 & CRASSULA_HELMSII_UK_REF_4 & 2.673 \\
\hline CRASSULA_BLIND_TEST_14 & CRASSULA_HELMSII_VICTORIA_REF_4 & 2.710 & CRASSULA_HELMSII_VICTORIA_REF_1 & 2.577 \\
\hline CRASSULA_BLIND_TEST_15 & CRASSULA_HELMSII_BELGIUM_REF_4 & 2.562 & CRASSULA_HELMSII_BELGIUM_REF_6 & 2.555 \\
\hline CRASSULA_BLIND_TEST_16 & CRASSULA_AQUATICA_REF_2 & 2.522 & CRASSULA_AQUATICA_REF_5 & 2.519 \\
\hline CRASSULA_BLIND_TEST_17 & CRASSULA_HELMSII_FLINDERS_ISLAND_REF_2 & 2.770 & CRASSULA_HELMSII_FLINDERS_ISLAND_REF_6 & 2.676 \\
\hline CRASSULA_BLIND_TEST_18 & CRASSULA_HELMSII_UK_REF_2 & 2.543 & CRASSULA_HELMSII_UK_REF_3 & 2.514 \\
\hline CRASSULA_BLIND_TEST_19 & CRASSULA_HELMSII_VICTORIA_REF_2 & 2.649 & CRASSULA_HELMSII_VICTORIA_REF_5 & 2.551 \\
\hline CRASSULA_BLIND_TEST_20 & CRASSULA_HELMSII_BELGIUM_REF_5 & 2.704 & CRASSULA_HELMSII_BELGIUM_REF_3 & 2.688 \\
\hline CRASSULA_BLIND_TEST_21 & CRASSULA_HELMSII_BELGIUM_REF_2 & 2.614 & CRASSULA_HELMSII_BELGIUM_REF_5 & 2.566 \\
\hline CRASSULA_BLIND_TEST_22 & CRASSULA_HELMSII_FLINDERS_ISLAND_REF_1 & 2.577 & CRASSULA_HELMSII_FLINDERS_ISLAND_REF_2 & 2.428 \\
\hline CRASSULA_BLIND_TEST_23 & CRASSULA_HELMSII_UK_REF_4 & 2.673 & CRASSULA_HELMSII_BELGIUM_REF_5 & 2.626 \\
\hline CRASSULA_BLIND_TEST_24 & CRASSULA_HELMSII_VICTORIA_REF_4 & 2.716 & CRASSULA_HELMSII_VICTORIA_REF_3 & 2.641 \\
\hline CRASSULA_BLIND_TEST_25 & CRASSULA_HELMSII_VICTORIA_REF_4 & 2.706 & CRASSULA_HELMSII_VICTORIA_REF_1 & 2.617 \\
\hline CRASSULA_BLIND_TEST_26 & CRASSULA_AQUATICA_REF_5 & 2.388 & CRASSULA_AQUATICA_REF_4 & 2.364 \\
\hline CRASSULA_BLIND_TEST_27 & CRASSULA_HELMSII_FLINDERS_ISLAND_REF_1 & 2.621 & CRASSULA_HELMSII_FLINDERS_ISLAND_REF_2 & 2.472 \\
\hline CRASSULA_BLIND_TEST_28 & CRASSULA_HELMSII_BELGIUM_REF_5 & 2.696 & CRASSULA_HELMSII_BELGIUM_REF_3 & 2.687 \\
\hline CRASSULA_BLIND_TEST_29 & CRASSULA_HELMSII_UK_REF_4 & 2.630 & CRASSULA_HELMSII_UK_REF_1 & 2.609 \\
\hline CRASSULA_BLIND_TEST_30 & CRASSULA_AQUATICA_REF_5 & 2.614 & CRASSULA_AQUATICA_REF_4 & 2.605 \\
\hline
\end{tabular}


Table S3. Identification results for rubbervine samples.

\begin{tabular}{|c|c|c|c|c|c|}
\hline Blind-test sample & Best match & Bruker Score & Second-best match & Bruker score & Identification call \\
\hline BLIND_TEST_17 & BRAZIL_PLANT_2_LEAF_1_REP_1 & 2.765 & BRAZIL_PLANT_2_LEAF_1_REP_2 & 2.692 & Brazil \\
\hline BLIND_TEST_18 & AUSTRALIA_PLANT_1_LEAF_1_REP_1 & 2.712 & AUSTRALIA_PLANT_1_LEAF_1_REP_2 & 2.698 & Australia \\
\hline BLIND_TEST_19 & BRAZIL_PLANT_1_LEAF_1_REP_2 & 2.677 & BRAZIL_PLANT_1_LEAF_1_REP_1 & 2.671 & Brazil \\
\hline BLIND_TEST_20 & AUSTRALIA_PLANT_1_LEAF_1_REP_1 & 2.664 & AUSTRALIA_PLANT_1_LEAF_1_REP_2 & 2.614 & Australia \\
\hline BLIND_TEST_21 & BRAZIL_PLANT_1_LEAF_2_REP_2 & 2.669 & BRAZIL_PLANT_1_LEAF_2_REP_1 & 2.615 & Brazil \\
\hline BLIND_TEST_22 & AUSTRALIA_PLANT_1_LEAF_2_REP_1 & 2.668 & AUSTRALIA_PLANT_1_LEAF_2_REP_2 & 2.557 & Australia \\
\hline BLIND_TEST_23 & AUSTRALIA_PLANT_1_LEAF_2_REP_1 & 2.700 & AUSTRALIA_PLANT_1_LEAF_2_REP_2 & 2.658 & Australia \\
\hline BLIND_TEST_24 & BRAZIL_PLANT_1_LEAF_2_REP_2 & 2.596 & BRAZIL_PLANT_1_LEAF_2_REP_1 & 2.580 & Brazil \\
\hline BLIND_TEST_25 & BRAZIL_PLANT_2_LEAF_1_REP_1 & 2.686 & BRAZIL_PLANT_2_LEAF_1_REP_2 & 2.584 & Brazil \\
\hline BLIND_TEST_26 & AUSTRALIA_PLANT_2_LEAF_2_REP_2 & 2.632 & AUSTRALIA_PLANT_2_LEAF_1_REP_1 & 2.606 & Australia \\
\hline BLIND_TEST_27 & BRAZIL_PLANT_1_LEAF_1_REP_2 & 2.574 & BRAZIL_PLANT_2_LEAF_2_REP_2 & 2.570 & Brazil \\
\hline BLIND_TEST_28 & AUSTRALIA_PLANT_2_LEAF_1_REP_1 & 2.684 & AUSTRALIA_PLANT_2_LEAF_2_REP_2 & 2.667 & Australia \\
\hline BLIND_TEST_29 & AUSTRALIA_PLANT_2_LEAF_2_REP_2 & 2.695 & AUSTRALIA_PLANT_2_LEAF_1_REP_1 & 2.645 & Australia \\
\hline BLIND_TEST_30 & BRAZIL_PLANT_1_LEAF_1_REP_2 & 2.560 & BRAZIL_PLANT_2_LEAF_1_REP_2 & 2.546 & Brazil \\
\hline BLIND_TEST_31 & AUSTRALIA_PLANT_2_LEAF_2_REP_2 & 2.643 & AUSTRALIA_PLANT_2_LEAF_2_REP_1 & 2.589 & Australia \\
\hline BLIND_TEST_32 & BRAZIL_PLANT_2_LEAF_2_REP_1 & 2.635 & BRAZIL_PLANT_1_LEAF_1_REP_2 & 2.609 & Brazil \\
\hline
\end{tabular}


Table S4. Old (1960s) and new (2010s) dried Sphingidae sample details.

\begin{tabular}{|c|c|c|}
\hline Sample $^{\mathrm{a}}$ & Details & Identification \\
\hline MJWC_106 & [UK] Somerset, Bruton, MVL, 2.vii.1966 [M.J.W. Cock] MJWC 2015-012 & Laothoe populi (Linnaeus) \\
\hline MJWC_107 & [UK] Somerset, Bruton, MVL, 22.v.1966 [M.J.W. Cock] MJWC 2015-008 & Smerinthus ocellata (Linnaeus) \\
\hline MJWC_108 & [UK] Somerset, Bruton, MVL, 7.v.1966 [M.J.W. Cock] & Deilephila elpenor (Linnaeus) \\
\hline MJWC_109 & UK, Ceredigion, Llanon, Brynamlwg, MVL trap, 30.v.2012, M.J.W. Cock & Laothoe populi (Linnaeus) \\
\hline MJWC_110 & UK, Ceredigion, Llanon, Brynamlwg, MVL trap, 19.v.2017, M.J.W. Cock & Smerinthus ocellata (Linnaeus) \\
\hline MJWC_111 & UK, Ceredigion, Llanon, Brynamlwg, MVL trap, 6.vii.2013, M.J.W. Cock & Deilephila elpenor (Linnaeus) \\
\hline MJWC_112 & [UK] Somerset, Bruton, MVL, 22.v.1966 [M.J.W. Cock] MJWC 2015-008 & Smerinthus ocellata (Linnaeus) \\
\hline MJWC_113 & UK, Ceredigion, Llanon, Brynamlwg, MVL trap, 30.v.2012, M.J.W. Cock & Laothoe populi (Linnaeus) \\
\hline MJWC_114 & UK, Ceredigion, Llanon, Brynamlwg, MVL trap, 6.vii.2013, M.J.W. Cock & Deilephila elpenor (Linnaeus) \\
\hline MJWC_115 & [UK] Somerset, Bruton, MVL, 2.vii.1966 [M.J.W. Cock] MJWC 2015-012 & Laothoe populi (Linnaeus) \\
\hline MJWC_116 & [UK] Somerset, Bruton, MVL, 7.v.1966 [M.J.W. Cock] & Deilephila elpenor (Linnaeus) \\
\hline MJWC_117 & UK, Ceredigion, Llanon, Brynamlwg, MVL trap, 19.v.2017, M.J.W. Cock & Smerinthus ocellata (Linnaeus) \\
\hline MJWC_118 & UK, Ceredigion, Llanon, Brynamlwg, MVL trap, 6.vii.2013, M.J.W. Cock & Deilephila elpenor (Linnaeus) \\
\hline MJWC_119 & UK, Ceredigion, Llanon, Brynamlwg, MVL trap, 19.v.2017, M.J.W. Cock & Smerinthus ocellata (Linnaeus) \\
\hline MJWC_120 & UK, Ceredigion, Llanon, Brynamlwg, MVL trap, 30.v.2012, M.J.W. Cock & Laothoe populi (Linnaeus) \\
\hline MJWC_121 & [UK] Somerset, Bruton, MVL, 7.v.1966 [M.J.W. Cock] & Deilephila elpenor (Linnaeus) \\
\hline MJWC_122 & [UK] Somerset, Bruton, MVL, 22.v.1966 [M.J.W. Cock] MJWC 2015-008 & Smerinthus ocellata (Linnaeus) \\
\hline MJWC_123 & [UK] Somerset, Bruton, MVL, 2.vii.1966 [M.J.W. Cock] MJWC 2015-012 & Laothoe populi (Linnaeus) \\
\hline MJWC_124 & [UK] Somerset, Bruton, MVL, 7.v.1966 [M.J.W. Cock] & Deilephila elpenor (Linnaeus) \\
\hline MJWC_125 & UK, Ceredigion, Llanon, Brynamlwg, MVL trap, 19.v.2017, M.J.W. Cock & Smerinthus ocellata (Linnaeus) \\
\hline MJWC_126 & [UK] Somerset, Bruton, MVL, 2.vii.1966 [M.J.W. Cock] MJWC 2015-012 & Laothoe populi (Linnaeus) \\
\hline MJWC_127 & UK, Ceredigion, Llanon, Brynamlwg, MVL trap, 6.vii.2013, M.J.W. Cock & Deilephila elpenor (Linnaeus) \\
\hline MJWC_128 & UK, Ceredigion, Llanon, Brynamlwg, MVL trap, 30.v.2012, M.J.W. Cock & Laothoe populi (Linnaeus) \\
\hline MJWC_129 & [UK] Somerset, Bruton, MVL, 22.v.1966 [M.J.W. Cock] MJWC 2015-008 & Smerinthus ocellata (Linnaeus) \\
\hline MJWC_130 & [UK] Somerset, Bruton, MVL, 22.v.1966 [M.J.W. Cock] MJWC 2015-008 & Smerinthus ocellata (Linnaeus) \\
\hline MJWC_131 & UK, Ceredigion, Llanon, Brynamlwg, MVL trap, 19.v.2017, M.J.W. Cock & Smerinthus ocellata (Linnaeus) \\
\hline MJWC_132 & UK, Ceredigion, Llanon, Brynamlwg, MVL trap, 6.vii.2013, M.J.W. Cock & Deilephila elpenor (Linnaeus) \\
\hline MJWC_133 & UK, Ceredigion, Llanon, Brynamlwg, MVL trap, 30.v.2012, M.J.W. Cock & Laothoe populi (Linnaeus) \\
\hline MJWC_134 & [UK] Somerset, Bruton, MVL, 7.v.1966 [M.J.W. Cock] & Deilephila elpenor (Linnaeus) \\
\hline
\end{tabular}

${ }^{a}$ Samples comprised a single leg from each source specimen, divided into five portions (information in square brackets was not included on the original data labels of the source specimens). 
Table S5. Identification results for Sphingidae samples.

(A) Old test samples against old-sample database entries

\begin{tabular}{|c|c|c|c|c|}
\hline Analyte & Best match & Bruker score & Second-best match & Bruker score \\
\hline MJWC_106 (Laothoe populi 1966) & MJWC_123 (Laothoe populi 1966) & 2.075 & MJWC_126 (Laothoe populi 1966) & 1.988 \\
\hline MJWC_107 (Smerinthus ocellata 1966) & MJWC_112 (Smerinthus ocellata 1966) & 2.134 & MJWC_122 (Smerinthus ocellata 1966) & 1.969 \\
\hline MJWC_108 (Deilephila elpenor 1966) & MJWC_121 (Deilephila elpenor 1966) & 2.425 & MJWC_116 (Deilephila elpenor 1966) & 2.402 \\
\hline MJWC_112 (Smerinthus ocellata 1966) & MJWC_107 (Smerinthus ocellata 1966) & 2.122 & MJWC_122 (Smerinthus ocellata 1966) & 2.053 \\
\hline MJWC_116 (Deilephila elpenor 1966) & MJWC_121 (Deilephila elpenor 1966) & 2.502 & MJWC_108 (Deilephila elpenor 1966) & 2.309 \\
\hline MJWC_121 (Deilephila elpenor 1966) & MJWC_116 (Deilephila elpenor 1966) & 2.388 & MJWC_108 (Deilephila elpenor 1966) & 2.363 \\
\hline MJWC_122 (Smerinthus ocellata 1966) & MJWC_112 (Smerinthus ocellata 1966) & 2.057 & MJWC_107 (Smerinthus ocellata 1966) & 1.932 \\
\hline MJWC_123 (Laothoe populi 1966) & MJWC_126 (Laothoe populi 1966) & 2.292 & MJWC_106 (Laothoe populi 1966) & 2.026 \\
\hline MJWC_124 (Deilephila elpenor 1966) & MJWC_134 (Deilephila elpenor 1966) & 2.301 & MJWC_121 (Deilephila elpenor 1966) & 2.063 \\
\hline MJWC_126 (Laothoe populi 1966) & MJWC_123 (Laothoe populi 1966) & 2.284 & MJWC_106 (Laothoe populi 1966) & 2.006 \\
\hline MJWC_129 (Smerinthus ocellata 1966) & No reliable identification & 1.342 & No reliable identification & 0.998 \\
\hline MJWC_130 (Smerinthus ocellata 1966) & No reliable identification & 1.369 & No reliable identification & 1.329 \\
\hline MJWC_134 (Deilephila elpenor 1966) & MJWC_124 (Deilephila elpenor 1966) & 2.207 & MJWC_121 (Deilephila elpenor 1966) & 2.175 \\
\hline
\end{tabular}

(B) New test samples against old-sample database entries

\begin{tabular}{|c|c|c|c|c|}
\hline Analyte & $\begin{array}{l}\text { Best } \\
\text { match }\end{array}$ & $\begin{array}{l}\text { Bruker } \\
\text { score }\end{array}$ & $\begin{array}{l}\text { Second-best } \\
\text { match }\end{array}$ & $\begin{array}{l}\text { Bruker } \\
\text { score }\end{array}$ \\
\hline MJWC_109 (Laothoe populi 2012) & MJWC_126 (Laothoe populi 1966) & 1.829 & MJWC_106 (Laothoe populi 1966) & 1.776 \\
\hline MJWC_110 (Smerinthus ocellata 2017) & No reliable identification & 1.177 & No reliable identification & 1.149 \\
\hline MJWC_111 (Deilephila elpenor 2013) & No reliable identification & 1.632 & No reliable identification & 1.549 \\
\hline MJWC_113 (Laothoe populi 2012) & MJWC_106 (Laothoe populi 1966) & 1.892 & MJWC_123 (Laothoe populi 1966) & 1.759 \\
\hline MJWC_114 (Deilephila elpenor 2013) & No reliable identification & 1.490 & No reliable identification & 1.486 \\
\hline MJWC_117 (Smerinthus ocellata 2017) & No reliable identification & 1.576 & No reliable identification & 1.239 \\
\hline MJWC_118 (Deilephila elpenor 2013) & No reliable identification & 1.499 & No reliable identification & 1.305 \\
\hline MJWC_119 (Smerinthus ocellata 2017) & No reliable identification & 1.639 & No reliable identification & 1.343 \\
\hline MJWC_120 (Laothoe populi 2012) & MJWC_123 (Laothoe populi 1966) & 1.914 & MJWC_126 (Laothoe populi 1966) & 1.845 \\
\hline MJWC_125 (Smerinthus ocellata 2017) & No reliable identification & 1.512 & No reliable identification & 1.305 \\
\hline MJWC_127 (Deilephila elpenor 2013) & No reliable identification & 1.694 & No reliable identification & 1.633 \\
\hline MJWC_128 (Laothoe populi 2012) & No reliable identification & 1.694 & No reliable identification & 1.594 \\
\hline MJWC_131 (Smerinthus ocellata 2017) & No reliable identification & 1.690 & No reliable identification & 1.396 \\
\hline MJWC_132 (Deilephila elpenor 2013) & No reliable identification & 1.624 & No reliable identification & 1.599 \\
\hline MJWC_133 (Laothoe populi 2012) & No reliable identification & 1.512 & No reliable identification & 1.491 \\
\hline
\end{tabular}


(C) New test samples against new-sample database entries

\begin{tabular}{lllll}
\hline Analyte & Best match & Bruker score & Second-best match & Bruker score \\
\hline MJWC_109 (Laothoe populi 2012) & MJWC_120 (Laothoe populi 2012) & 2.385 & MJWC_128 (Laothoe populi 2012) & 2.378 \\
MJWC_110 (Smerinthus ocellata 2017) & MJWC_117 (Smerinthus ocellata 2017) & 2.293 & MJWC_119 (Smerinthus ocellata 2017) & 2.028 \\
MJWC_111 (Deilephila elpenor 2013) & MJWC_132 (Deilephila elpenor 2013) & 2.326 & MJWC_118 (Deilephila elpenor 2013) & 2.308 \\
MJWC_113 (Laothoe populi 2012) & MJWC_128 (Laothoe populi 2012) & 2.528 & MJWC_133 (Laothoe populi 2012) & 2.453 \\
MJWC_114 (Deilephila elpenor 2013) & MJWC_127 (Deilephila elpenor 2013) & 2.248 & MJWC_111 (Deilephila elpenor 2013) & 2.180 \\
MJWC_117 (Smerinthus ocellata 2017) & MJWC_131 (Smerinthus ocellata 2017) & 2.465 & MJWC_110 (Smerinthus ocellata 2017) & 2.371 \\
MJWC_118 (Deilephila elpenor 2013) & MJWC_111 (Deilephila elpenor 2013) & 2.382 & MJWC_127 (Deilephila elpenor 2013) & 2.256 \\
MJWC_119 (Smerinthus ocellata 2017) & MJWC_125 (Smerinthus ocellata 2017) & 2.507 & MJWC_131 (Smerinthus ocellata 2017) & 2.352 \\
MJWC_120 (Laothoe populi 2012) & MJWC_109 (Laothoe populi 2012) & 2.287 & MJWC_113 (Laothoe populi 2012) & 2.088 \\
MJWC_125 (Smerinthus ocellata 2017) & MJWC_119 (Smerinthus ocellata 2017) & 2.591 & MJWC_131 (Smerinthus ocellata 2017) & 2.141 \\
MJWC_127 (Deilephila elpenor 2013) & MJWC_132 (Deilephila elpenor 2013) & 2.461 & MJWC_111 (Deilephila elpenor 2013) & 2.338 \\
MJWC_128 (Laothoe populi 2012) & MJWC_133 (Laothoe populi 2012) & 2.475 & MJWC_109 (Laothoe populi 2012) & 2.420 \\
MJWC_131 (Smerinthus ocellata 2017) & MJWC_117 (Smerinthus ocellata 2017) & 2.517 & MJWC_119 (Smerinthus ocellata 2017) & 2.315 \\
MJWC_132 (Deilephila elpenor 2013) & MJWC_127 (Deilephila elpenor 2013) & 2.577 & MJWC_111 (Deilephila elpenor 2013) & 2.393 \\
MJWC_133 (Laothoe populi 2012) & MJWC_128 (Laothoe populi 2012) & 2.437 & MJWC_113 (Laothoe populi 2012) & 2.422 \\
\hline
\end{tabular}

(D) Old test samples against new-sample database entries

\begin{tabular}{lllll}
\hline Analyte & Best match & Bruker & Becond-best match & Bruker \\
& & score & & score \\
\hline MJWC_106 (Laothoe populi 1966) & MJWC_113 (Laothoe populi 2012) & 1.882 & MJWC_109 (Laothoe populi 2012) & 1.797 \\
MJWC_107 (Smerinthus ocellata 1966) & No reliable identification & 1.527 & No reliable identification & 1.372 \\
MJWC_108 (Deilephila elpenor 1966) & No reliable identification & 1.476 & No reliable identification & 1.430 \\
MJWC_112 (Smerinthus ocellata 1966) & MJWC_131 (Smerinthus ocellata 2017) & 1.734 & No reliable identification & 1.553 \\
MJWC_115 (Laothoe populi 1966) & MJWC_109 (Laothoe populi 2012) & 1.715 & No reliable identification & 1.679 \\
MJWC_116 (Deilephila elpenor 1966) & No reliable identification & 1.544 & No reliable identification & 1.438 \\
MJWC_121 (Deilephila elpenor 1966) & MJWC_132 (Deilephila elpenor 2013) & 1.715 & No reliable identification & 1.623 \\
MJWC_122 (Smerinthus ocellata 1966) & No reliable identification & 1.488 & No reliable identification & 1.361 \\
MJWC_123 (Laothoe populi 1966) & MJWC_120 (Laothoe populi 2012) & 1.884 & MJWC_109 (Laothoe populi 2012) & 1.848 \\
MJWC_124 (Deilephila elpenor 1966) & No reliable identification & 1.603 & No reliable identification & 1.547 \\
MJWC_126 (Laothoe populi 1966) & MJWC_120 (Laothoe populi 2012) & 1.959 & MJWC_109 (Laothoe populi 2012) & 1.909 \\
MJWC_129 (Smerinthus ocellata 1966) & No reliable identification & 0.936 & No reliable identification & 0.709 \\
MJWC_130 (Smerinthus ocellata 1966) & No reliable identification & 1.467 & No reliable identification & 1.245 \\
MJWC_134 (Deilephila elpenor 1966) & No reliable identification & 1.678 & No reliable identification & 1.569 \\
\hline
\end{tabular}

